\title{
Exome Sequencing Landscape Analysis in Ovarian Clear Cell Carcinoma Shed Light on Key Chromosomal Regions and Mutation Gene Networks
}

\author{
Ryusuke Murakami, ${ }^{*}$ Noriomi Matsumura, ${ }^{* \dagger}$ J.B. Brown, ${ }^{\ddagger}$ Koichiro Higasa, ${ }^{\complement}$ Takanobu Tsutsumi, ${ }^{\llbracket}$ Mayumi Kamada,
} Hisham Abou-Taleb, ${ }^{*}$ Yuko Hosoe, ${ }^{*}$ Sachiko Kitamura, ${ }^{*}$ Ken Yamaguchi, ${ }^{*}$ Kaoru Abiko, ${ }^{*}$ Junzo Hamanishi, ${ }^{*}$ Tsukasa Baba, ${ }^{*}$ Masafumi Koshiyama, ${ }^{*}$ Yasushi Okuno, ${ }^{\S}$ Ryo Yamada, ${ }^{\oplus}$ Fumihiko Matsuda, ${ }^{\text {『 }}$ Ikuo Konishi, ${ }^{*}$ and Masaki Mandai ${ }^{* \dagger}$

From the Departments of Gynecology and Obstetrics* and Clinical System Onco-Informatics ${ }^{\S}$ and the Laboratory for Molecular Biosciences, ${ }^{\ddagger}$ Life Science Informatics Research Unit, Graduate School of Medicine, and The Center for Genomics Medicine, "Kyoto University, Kyoto; and the Department of Obstetrics and Gynecology, ${ }^{\dagger}$ Kindai University Faculty of Medicine, Osaka, Japan

Accepted for publication

June 8, 2017.

Address correspondence to Noriomi Matsumura, M.D., Ph.D., 54 Shogoin

Kawahara-cho, Sakyo-ku, Kyoto 606-8507, Japan. E-mail: noriomi@ kuhp.kyoto-u.ac.jp.

\begin{abstract}
Previous studies have reported genome-wide mutation profile analyses in ovarian clear cell carcinomas (OCCCs). This study aims to identify specific novel molecular alterations by combined analyses of somatic mutation and copy number variation. We performed whole exome sequencing of 39 OCCC samples with 16 matching blood tissue samples. Four hundred twenty-six genes had recurrent somatic mutations. Among the 39 samples, ARID1A (62\%) and PIK3CA (51\%) were frequently mutated, as were genes such as KRAS $(10 \%), P P P 2 R 1 A(10 \%)$, and PTEN (5\%), that have been reported in previous OCCC studies. We also detected mutations in MLL3 (15\%), ARID1B (10\%), and PIK3R1 (8\%), which are associations not previously reported. Gene interaction analysis and functional assessment revealed that mutated genes were clustered into groups pertaining to chromatin remodeling, cell proliferation, DNA repair and cell cycle checkpointing, and cytoskeletal organization. Copy number variation analysis identified frequent amplification in chr8q (64\%), chr20q (54\%), and chr17q (46\%) loci as well as deletion in chr19p (41\%), chr13q (28\%), chr9q (21\%), and chr18q (21\%) loci. Integration of the analyses uncovered that frequently mutated or amplified/deleted genes were involved in the KRAS/phosphatidylinositol 3-kinase $(82 \%)$ and MYC/retinoblastoma (75\%) pathways as well as the critical chromatin remodeling complex switch/sucrose nonfermentable (85\%). The individual and integrated analyses contribute details about the OCCC genomic landscape, which could lead to enhanced diagnostics and therapeutic options. (Am J Pathol 2017, 187: 2246-2258; http://dx.doi.org/10.1016/j.ajpath.2017.06.012)
\end{abstract}

Ovarian cancer is the poorest prognostic disease in gynecologic malignant tumors and is complicated by the fact that the prognoses and corresponding behaviors in histologic subtypes are distinct. ${ }^{1}$ High-grade serous ovarian cancer is the most frequent subtype, accounting for $68 \%$ of ovarian cancer. ${ }^{1}$ The ovarian clear cell carcinoma (OCCC) subtype is highly resistant to chemotherapy and often associated with endometriosis. ${ }^{2}$ The OCCC subtype accounts for approximately $4 \%$ to $12 \%$ of all epithelial ovarian carcinomas in European and North American countries, whereas its prevalence increases to $15 \%$ to $20 \%$ in Japan. ${ }^{3,4}$ To date, only eight OCCC samples have been analyzed by exome sequencing, ${ }^{5}$ which is an extremely small sample size compared with the 316 high-grade serous ovarian cancer samples analyzed by The Cancer Genome Atlas. ${ }^{6}$ Hence, the genomic landscape of the rarer OCCC subtype remains insufficiently investigated on a larger scale.

Thus far, OCCC-specific somatic mutations in focused target-level studies of $A R I D I A,,^{5,7}$ PIK3CA, ${ }^{5,8,9}$ and $P P P 2 R 1 A^{5,10}$ and copy number amplification of ZNF2 $17^{11,12}$ have been reported in Caucasian populations. Interestingly, these genetic alterations of the OCCC subtype are entirely different from the high-grade serous ovarian

Supported by The Ministry of Education, Culture, Sports, Science and Technology grants-in-aid for scientific research 25462590 (N.M.), 26253080 (I.K.), and 17H01587 (I.K.).

Disclosures: None declared. 
cancer subtype in which TP53 and BRCA alterations have been observed frequently. ${ }^{13}$

A pan-cancer study performed mutation and copy number analyses using large numbers of samples of various tumor types, where analyses grouped tumor types exclusively by mutation or copy number alteration. ${ }^{14}$ OCCC was not specifically analyzed as a tumor type. However, it is particularly interesting because suppression of a single tumor growth signaling mechanism via molecularly targeted chemotherapy leads to increased signaling in a different mechanism, ${ }^{15,16}$ and hence analysis of only mutations or only copy number alterations alone is insufficient. A thorough investigation of both independent mutation and copy number analyses as well as a joint, integrated analysis for OCCC that clarifies the separate and joint landscapes has yet to be reported. Therefore, we aim to clarify the patterns of genomic alterations in OCCC and to identify whether such alterations are caused by mutation addiction, copy number addiction, or both.

Until recently, single-nucleotide polymorphism (SNP) array technology has been used for differential analysis of patients, and it is still a cost-effective competing technology to newer next-generation sequencing. ${ }^{17}$ In the case of OCCC, samples and data are highly limited; standard regions and hotspots available in SNP arrays may not sufficiently cover the subtype-specific alterations that explain the disease. The resolution of whole exome sequencing circumvents these shortcomings and is applicable for the detection of novel OCCC-specific mutation elements.

In this study, we applied whole-exome sequencing to 39 OCCC samples, the largest OCCC-specific collection to our knowledge, and to 16 noncancerous blood tissue samples provided by a subset of the same patients. To elucidate altered genetic elements, we compared the tumor-blood sequencing results and additionally profiled the tumor samples against a public database of 1208 healthy Japanese exomes. ${ }^{18}$ In addition, copy number analysis of these OCCC exome sequencing data sets was undertaken. Critically, we integrated the independent single-nucleotide variant (SNV) and copy number variant (CNV) analyses, aiming to improve the resolution of the molecular profiling of OCCC, such as identifying the links between functional subnetworks of genes with molecular alterations.

\section{Materials and Methods}

\section{Samples}

Ovarian tumor samples were collected from 39 patients with OCCC who had no pretreatments and underwent surgery between 1997 and 2013 at Kyoto University Hospital. This study was approved by an institutional ethics committee in which donors gave written informed consent in accordance with institutional and national guidelines. DNA was extracted from the 39 frozen tumor tissues stored at $-80^{\circ} \mathrm{C}$ and from matching blood lymphocyte samples available from 16 of the 39 patients. During the initial years of sample collection, it was not assumed that samples were going to be used for exome comparison, and consequently, blood lymphocyte samples could be not extracted from 23 of the 39 patients because of a lack of patient consent. The genomic DNAs were purified using QIAamp DNA Mini Kits (Qiagen, Hilden, Germany) following the manufacturer's protocol. Quality of DNA was assessed by the DNA concentration $(>100 \mathrm{ng} / \mu \mathrm{L})$ and electrophoresis. In total, 3 $\mu \mathrm{g}$ of genomic DNA was used for exome sequencing.

\section{Exome Sequencing}

Whole exome sequencing was performed using Agilent SureSelect Human All Exon V5 + UTRs (Agilent Technologies, Santa Clara, CA). Exome sequencing data were generated by 100-base paired-end reads on an Illumina HiSeq 2000 system (Illumina Inc., San Diego, CA) and analyzed through an in-house pipeline. Paired-end reads in FASTQ files were mapped on to the human reference genome sequence (GCRh37/hg19) with Burrows-Wheeler Aligner (BWA) software version 0.7.10 (http://bio-bwa.sourceforge.net). ${ }^{19}$ The output files in SAM format were converted into sorted BAM files, and duplicate reads were removed using SAMtools version 1.0 (http://samtools.sourceforge.net). ${ }^{20}$ Downstream steps, including insertion/deletion realignment, recalibration of base quality, variant quality score recalibration, and variant calling, were performed with the Genome Analysis Toolkit version 3.2. ${ }^{21}$ ANNOVAR version 2014Jul14 ${ }^{22}$ was used to annotate variants in variant call format files. Mean coverage of target regions was $92.6 \times$ (range, $68.8 \times$ to $122.7 \times$ ), and a mean of $92 \%$ of target regions were covered with at least $20 \times$ coverage (range, $87 \%$ to $96 \%$ ). A total of 4620 variants were left for subsequent analyses after filtering out variants with quality scores $<40$ or with sample call rates $<95 \%$ (52 of 55 samples) to avoid misidentification of mutations occurring in OCCC samples. Samples were further filtered as explained below (Figure 1).

\section{Sanger Sequencing}

Mutations in specific genes were confirmed by Sanger sequencing (Table 1). Ten nanograms of genomic DNA was mixed in a total $50-\mu \mathrm{L}$ reaction that contained $25 \mu \mathrm{L}$ of Emerald Amp MAX PCR Master Mix (Takara Bio Inc., Kusatsu, Shiga, Japan), $1 \mu \mathrm{L}(100 \mathrm{pmol} / \mu \mathrm{L})$ of forward primer, and $1 \mu \mathrm{L}(100 \mathrm{pmol} / \mu \mathrm{L})$ of reverse primer in a PCR tube. PCR amplification was performed using a C1000 Touch Thermal Cycler (Bio-Rad, Hercules, CA) by the following procedure: denaturation at $98^{\circ} \mathrm{C}$ for 5 minutes; 30 cycles of the following three per-cycle conditions of $98^{\circ} \mathrm{C}$ for 10 seconds, $55^{\circ} \mathrm{C}$ for 30 seconds, and $72^{\circ} \mathrm{C}$ for 1 minute; subsequent holding at $72^{\circ} \mathrm{C}$ for 1 minute; and finally incubation at $12^{\circ} \mathrm{C}$. The primer specificity and optimal cycling conditions were verified by detecting single-band amplicons of the PCR products. The PCR products were purified with 


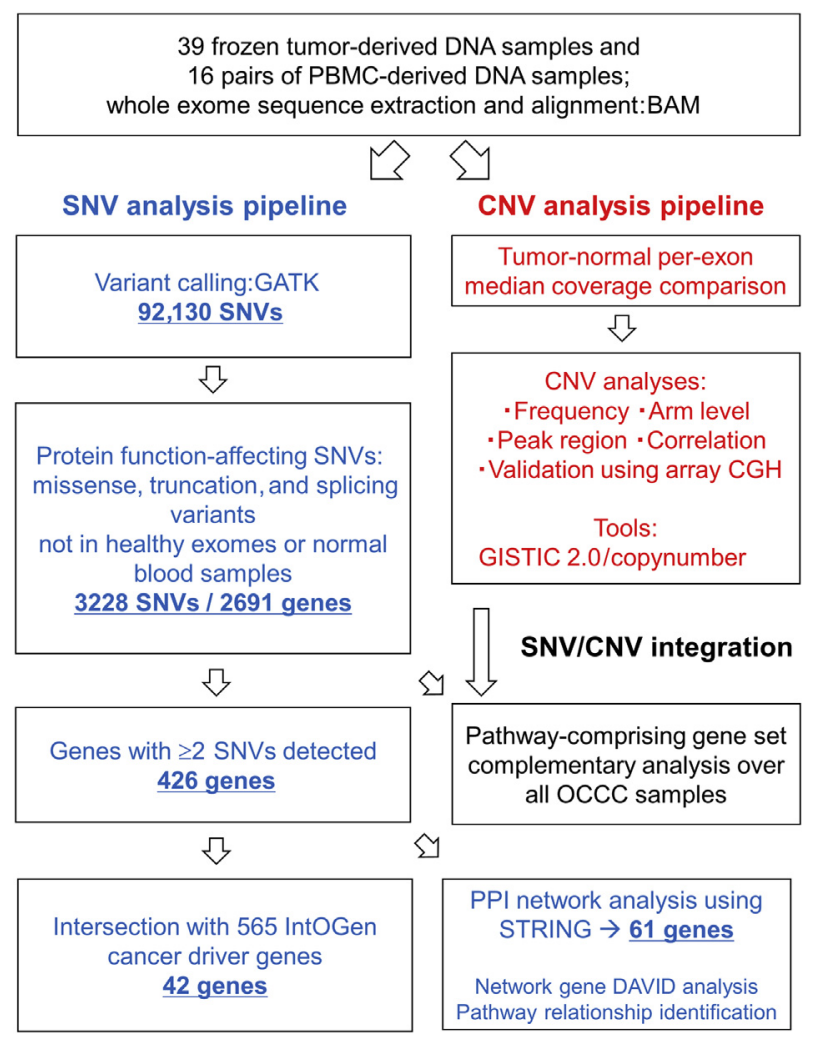

Figure 1 Workflow for detection of somatically mutated genes and copy number alterations in ovarian clear cell carcinoma (OCCC) samples. An initial pool of somatic mutations was obtained from exome sequencing data of tumors by comparing with their normal counterparts. These mutations were further refined by using functional annotation and variants found in public and private exome sequencing data from healthy individuals. A list of genes that had at least two remaining somatic mutations after filtering was intersected with known cancer driver genes to extract reliable OCCCassociated genes. For copy number analysis, median coverages of each exon were compared between tumor and normal tissues. For the samples without normal counterparts, the mean coverage from the other 16 patients was used as a surrogate. Copy number alterations were assessed at peak regions, at the gene level, at the arm level, and for genome-wide correlations. CGH, comparative genomic hybridization; CNV, copy number variation; DAVID, Database for Annotation, Visualization and Integrated Discovery; GATK, Genome Analysis Toolkit; PBMC, peripheral blood mononuclear cell; PPI, protein-protein interaction.

QIAquick PCR Purification Kit (Qiagen), and the $0.3-\mu \mathrm{L}$ DNA template $(10 \mathrm{ng})$ with $3.2 \mu \mathrm{L}$ of primer $(100 \mathrm{pmol} / \mu \mathrm{L})$ was run on the 3130xl Genetic Analyzer (Thermo Fisher Scientific/Applied Biosystems, Waltham, MA).

\section{Somatic Mutation Gene Analysis}

Functional mutations [ie, missense, truncation (nonsense variants and indels in coding regions), and splicing variants] were extracted after filtering out the variants found in exome sequence data sets from at least one of the following: normal tissue counterparts of the 16 patients with OCCC and/or 1208 healthy Japanese individuals in the Human Genetic Variation Database version 1.0 (http://www.hgvd.genome.med.kyoto-u.ac.jp) or 338 cancer-free Japanese individuals in an in-house database. This application of multiple criteria serves as a quality control check and a method to incorporate as many tumors as possible into the analysis, even if matched normal samples are not available for reasons mentioned above. Therefore, although strict tumor-normal matched pair criteria for the definition of somatic mutation cannot be applied, our quality controls provide at least a basis for rationalizing that the mutations are likely so. Along this line, the mutations detected were qualified as somatic.

A list totaling 565 unique known driver genes was obtained from the IntOGen Mutational Cancer Driver Genes database release 2012.12 23,24 (https://www.intogen.org), and the Pan-cancer study. ${ }^{25}$

MutSigCV version $1.4^{26}$ was applied for detecting genes that had functional somatic mutations more frequently than statistical expectation in the 39 OCCC tumor samples.

MutationMapper version 1.0.1 developed by cBioPortal (Memorial Sloan Kettering Cancer Center, New York, NY; http://www.cbioportal.org/tools.jsp $)^{27}$ was used for visualization of distribution patterns of the somatic mutations identified in PIK3CA and ARIDIA.

\section{Copy Number Analysis}

Median coverages of each exon were calculated, and those from tumor and normal tissues were compared for copy number analysis. To analyze the 23 samples for which corresponding normal tissues were not available, the mean coverage of the exome sequencing data from normal tissues of the other 16 patients was used as a surrogate coverage value. Copy number value was generated with transformed $\log _{2}$ ratios of tumor/ normal coverages after applying a moving mean of 60 exons. As a validation set, an array comparative genomic hybridization (CGH) data set obtained from 117 OCCC cases with $\log _{2}$ normalized data (GSE58342) ${ }^{28}$ was downloaded from the Gene Expression Omnibus (https://www.ncbi.nlm. nih.gov/geo; accession number GSE58342). Five array CGH samples were excluded from the analysis because of duplication. Bioconductor package copynumber, ${ }^{29}$ an extension to R software version 3.1.2 (The R Foundation for Statistical Computing, Vienna, Austria; http://www.r-project. org), was used for analyses and visualization of CNVs, where $\mathrm{CNV}$ was determined by the $\log _{2}$ ratio having a value of $>0.25$ for amplification and $<-0.25$ for deletion in a single segmentation analysis. ${ }^{28,30-32}$

GISTIC version 2.0 (The Broad Institute, Boston, MA; http://archive.broadinstitute.org/cancer/cga/gistic) analysis was performed for identifying copy number amplifications and deletions at the arm level, as well as at peak loci and gene levels in the two data sets. ${ }^{33}$ A threshold for variation was set to 0.25 (Kyoto) or 0.15 (GSE58342) of the absolute copy number $\log _{2}$ scaled values. The correlations of peak and arm-level amplifications and deletions found in the two data sets were closely examined. 


\section{Pathway Analysis}

Protein-protein interaction network analysis of genes retained after somatic mutation filtering (Figure 1) was performed using STRING version 10 (http://www.stringdb.org). ${ }^{34}$

Candidate somatic mutations were mapped onto a proteinprotein interaction network. Networks of less than five interactions were excluded. Network visualization was performed in Cytoscape software version 2 (http://www.cytoscape.org). ${ }^{35}$ Using genes in the extracted networks, pathway analyses with the Database for Annotation, Visualization and Integrated Discovery (DAVID) Bioinformatics Resources version 6.7 (NIH, Bethesda, MD; https://david.ncifcrf.gov) were performed to annotate genes with functional roles. Nodes of the two networks were colored for functional roles by using the MultiColoredNodes plugin within Cytoscape. ${ }^{36}$

Integrated analysis of SNVs and CNVs was performed by focusing on the pathways enriched with genes found to be frequently altered in our analyses.

\section{Quality Assessment for Somatic Mutation Genes}

For evaluating the quality of mutation data from the cases with or without matched normal samples, the Fisher exact test was used for counts of variants between 16 paired cases and 23 unpaired cases using 426 candidate somatic mutation genes. Then we compared core somatic mutations listed in 42 IntOGen pan cancer genes and the 46 protein-protein interaction (PPI) network genes in each of the subgroups. Moreover, we examined variant allele frequency (VAF) as calculated by the variant allele percentage and total read counts, which represent copy number status, in truncating and missense mutations. Variants with $>30$ total reads were retained. The truncation mutation variants $(n=172)$ and missense mutation variants $(n=854)$ were examined. The most frequently mutated gene, ARIDIA in truncating mutations, and the gene with the highest frequency of missense mutations, PIK3CA, were visualized by scatterplot coloring for identification.

\section{Immunohistochemical Analysis for BAF250a Expression}

Immunohistochemical staining for BAF250a was performed and evaluated similarly to methods previously reported. $^{37}$ Briefly, protein expression was evaluated by determining the Immuno-Reactive Score (IRS) ranging from 0 to 300 , which is obtained by multiplying the percentage of positively stained cells by the intensity of the reaction, which can take the values $0,1,2$, or 3 . Scores between 0 and 49 were classified as negative, whereas scores $\geq 50$ were considered to indicate positive expression. The association between BAF250a expression and ARIDIA mutation status with or without ARIDIA deletion was examined.

\section{Immunohistochemical Analysis for PTEN Expression}

PTEN immunohistochemistry was performed with the PTEN monoclonal mouse antibody (clone 6H2.1; Dako Cytomation, Carpinteria, CA). Antigen retrieval was accomplished using EDTA buffer ( $\mathrm{pH}$ 9.0) with steaming for 15 minutes, followed by the PTEN antibody (dilution 1:150). Diaminobenzidine was used as the chromogen. For tumors with mutant PTEN and loss of PTEN protein expression, positively stained blood vessels and stromal cells were used as an internal positive control. An IRS ranged from 0 to 300, similar to BAF250a described above, with an identical cutoff of 50 .

\section{Comparison of Copy Number by Exome Sequencing versus SNP Array}

For the subset of 12 OCCC samples with matching SNP arrays downloaded from Gene Expression Omnibus (https:// www.ncbi.nlm.nih.gov/geo; accession number GSE30311), ${ }^{30}$ copy number analysis was performed using Bioconductor packages cn.farms ${ }^{38}$ and copynumber. ${ }^{29}$

\section{Statistical Analysis}

$\mathrm{R}$ version 3.1.2 was used for statistical analyses. The Fisher exact test, $t$-test, and Pearson correlation test were used where appropriate. $P$ values were adjusted to false discovery rate (FDR) q-values using the p.adjust function in $\mathrm{R}$ with parameter method $=\mathrm{fdr}$.

\section{Results}

\section{Identification of Somatic Mutations in OCCC}

In total, 92,130 variants were identified, containing 3228 functional variants after variants found in matching blood samples or in the aforementioned healthy exomes were filtered out; 2838 nonsynonymous (88\%), 279 truncation [143 stop gain/loss (5\%) and 136 in-/out-of-frame insertion/ deletion (4\%)], 66 nonframeshift insertion/deletion (2\%), and 45 splicing variants (1\%) were located in 2691 genes (Figure 1). Among a total of 3007 nonindel variants, $\mathrm{C}$ to $\mathrm{T}$, $\mathrm{T}$ to $\mathrm{C}, \mathrm{C}$ to $\mathrm{G}, \mathrm{C}$ to $\mathrm{A}, \mathrm{T}$ to $\mathrm{G}$, and $\mathrm{T}$ to $\mathrm{A}$ transitions accounted for $50 \%, 16 \%, 15 \%, 10 \%, 5 \%$, and $4 \%$ of changes, respectively (Figure 2, A and B).

A total of 426 genes had at least two somatic variants (Supplemental Table S1) in which 42 genes were included in a prominent list of cancer driver genes previously reported. $^{23-25}$ Nine of those 42 genes were mutated in $>10 \%$ of 39 samples, of which ARIDIA had the highest number of mutations (29 truncation and 1 missense) (Figure 2C). MutSigCV ${ }^{26,39}$ identified ARIDIA and $P I K 3 C A$ as significantly mutated genes in the tumor samples $(\mathrm{q}<0.0001)$. Twenty-four $(62 \%)$ and $20(51 \%)$ of 39 samples had respective mutations in ARIDIA or PIK3CA 
Table 1 Summary of Primers for Sanger Sequencing

\begin{tabular}{|c|c|c|c|}
\hline Chromosome & Position & Gene & Forward primer \\
\hline 1 & 27056213 & ARID1A & $5^{\prime}-\mathrm{ATTCAGTGGCCAGAGGCCAT-3^{ \prime }}$ \\
\hline 1 & 27056232 & ARID1A & $5^{\prime}$-ATTCAGTGGCCAGAGGCCAT-3' \\
\hline 1 & 27056319 & ARID1A & $5^{\prime}-\mathrm{ATTCAGTGGCCAGAGGCCAT-3^{ \prime }}$ \\
\hline 1 & 27057727 & ARID1A & $5^{\prime}$-АCTCATCATCAGTGCATAGC-3' \\
\hline 1 & 27057818 & ARID1A & $5^{\prime}$-АCTCATCATCAGTGCATAGC-3' \\
\hline 1 & 27058063 & ARID1A & $5^{\prime}$-ACTCATCATCAGTGCATAGC-3' \\
\hline 1 & 27092737 & ARID1A & 5'-AGTTCTAGTTTTGGGGACCC-3' \\
\hline 1 & 27092787 & ARID1A & 5'-AGTTCTAGTTTTGGGGACCC-3' \\
\hline 1 & 27093048 & ARID1A & 5'-AGTTCTAGTTTTGGGGACCC-3' \\
\hline 1 & 27099102 & ARID1A & 5'-CGTGTGTTTGTGTGAGAGTT-3' \\
\hline 1 & 27099325 & $A R I D 1 A$ & 5'-CGTGTGTTTGTGTGAGAGTT-3' \\
\hline 1 & 27101092 & ARID1A & $5^{\prime}$-CTTCCCCTCAGCAAGATGTAT-3' \\
\hline 1 & 27101099 & ARID1A & $5^{\prime}$-СTTCCCCTCAGCAAGATGTAT-3' \\
\hline 1 & 27101273 & ARID1A & $5^{\prime}$-CTTCCCCTCAGCAAGATGTAT-3' \\
\hline 1 & 27102196 & ARID1A & 5'-GACAGACAGCTTAGAAGCCA-3' \\
\hline 1 & 27105553 & $A R I D 1 A$ & 5'-TGCTCTGTGGAGAACCTTTG-3' \\
\hline 1 & 27106354 & ARID1A & $5^{\prime}$-AGAGTTCAGAGGCCATCAAG-3' \\
\hline 1 & 27106478 & ARID1A & $5^{\prime}$-AGAGTTCAGAGGCCATCAAG- $3^{\prime}$ \\
\hline 1 & 27106861 & ARID1A & 5'-AGAGTTCAGAGGCCATCAAG-3' \\
\hline 1 & 27107152 & ARID1A & 5'-CCGGAACATCAAGATCCTAG-3' \\
\hline 3 & 178927980 & PIK3CA & 5'-CTCATGCTTGCTTTGGTTCA-3' \\
\hline 3 & 178936082 & PIK3CA & 5'-GATTGGTTCTTTCCTGTCTCT-3' \\
\hline 6 & 157517352 & $A R I D 1 B$ & $5^{\prime}$-GACAGAGTGAGACTCCATCT-3' \\
\hline 6 & 157528310 & $A R I D 1 B$ & $5^{\prime}$-GTCCGTAGCTTGTCATTCGT-3' \\
\hline 19 & 11098500 & SMARCA4 & $\begin{array}{r}5^{\prime}-\text { AGAGCTCAACATGACGCCCT-3' } \\
\text { (table continues) }\end{array}$ \\
\hline
\end{tabular}

genes (Figure 2D). For the key PPP2RIA gene previously analyzed in ovarian and endometrial cancers, ${ }^{10}$ four of 39 samples $(10 \%)$ contained the p.R183W hot spot mutation. Chromatin modification-related KMT2C/MLL3 and chromatin remodeling-related $A R I D I B$ were identified for novel association with OCCC, having mutation rates of $15 \%$ and $10 \%$, respectively. We were also able to identify $K R A S$, $P I K 3 R 1$, and PTEN with considerable mutation frequencies of $10 \%, 8 \%$, and 5\%, respectively (Figure 2, C and D).

We further examined mutation patterns of the frequently mutated genes ARIDIA and PIK3CA. Across 20 cases of mutated $P I K 3 C A$, five missense mutations were identified, in which the p.H1047R hot spot mutation was observed in 11 cases (Figure 2E). In contrast, 29 of 30 mutations identified in $A R I D I A$ across 24 cases were truncating mutations.
The truncations were found dispersed throughout ARIDIA (Figure 2F).

A total of 55 of 56 somatic mutations (98\%) were validated by Sanger sequencing of ARIDIA (26/27), PIK3CA (19/19), PPP2RIA (4/4), PTEN (2/2), ARIDIB (3/3), and SMARCA4 (1/1) (Table 1).

\section{Identification of CNV and Correlations at Multiple} Scales

Averaged CNV signals across the 39 exome sequencing data and across 112 array CGH samples (GSE58342, overlapping OCCC samples excluded) are shown in Figure 3. The overall CNV patterns are highly concordant between these two independent data sets. Here, we focus on 
Table 1 (continued)

\begin{tabular}{|c|c|c|c|c|}
\hline Reverse primer & Verification & Reference sequence & Alternative sequence & Counts \\
\hline $5^{\prime}$-CACAATCACCATCTACCTGC-3' & Verified & G & GC & 1 \\
\hline $5^{\prime}$-САСААТСАССАТСТАССТGC-3' & Verified & G & $\mathrm{T}$ & 1 \\
\hline 5'-TGCTGTGTCGTCGACTGCTG-3' & Verified & C & $\mathrm{T}$ & 1 \\
\hline 5'-TGCTGTGTCGTCGACTGCTG-3' & Verified & CA & $\mathrm{C}$ & 1 \\
\hline 5'-TGCTGTGTCGTCGACTGCTG-3' & Verified & GC & G & 1 \\
\hline 5'-GGCTTCTTTAGCCCAATGAG-3' & Verified & $C$ & $T$ & 1 \\
\hline 5'-GGCTTCTTTAGCCCAATGAG-3' & Verified & $\mathrm{T}$ & $\mathrm{TA}$ & 1 \\
\hline 5'-AGACTATCTAGTCCGGTGTC-3' & Verified & CA & $\mathrm{C}$ & 1 \\
\hline $5^{\prime}$-CACTGTCATGCCAAGCAAAC-3' & Verified & $\mathrm{T}$ & TC & 1 \\
\hline $5^{\prime}$-CACTGTCATGCCAAGCAAAC- $3^{\prime}$ & Verified & C & $T$ & 2 \\
\hline 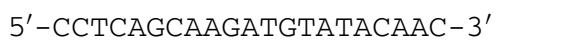 & Verified & G & GT & 1 \\
\hline $5^{\prime}$-CCTCAGCAAGATGTATACAAC- $3^{\prime}$ & Verified & C & $\mathrm{T}$ & 2 \\
\hline $5^{\prime}$-CCTCAGCAAGATGTATACAAC-3' & Not detected & C & $\mathrm{T}$ & 1 \\
\hline 5'-CATGGCGGGTAATGATGTCCCTCAA-3' & Verified & C & $\mathrm{T}$ & 1 \\
\hline 5'-CTTGTCCTTGCCTGAAAAGG-3' & Verified & C & $\mathrm{T}$ & 1 \\
\hline 5'-CAGCAGTACCACAGCCATCTC-3' & Verified & C & $\mathrm{T}$ & 1 \\
\hline $5^{\prime}$-CAGCAGTACCACAGCCATCTC- $3^{\prime}$ & Verified & CT & C & 1 \\
\hline $5^{\prime}$-CAGCAGTACCACAGCCATCTC- $3^{\prime}$ & Verified & C & $\mathrm{T}$ & 2 \\
\hline 5'-TCCCACGGCTGTCATGACTG-3' & Verified & G & $T$ & 1 \\
\hline $5^{\prime}$-AGAGAGAAGGTTTGACTGCC-3' & Verified & $\mathrm{T}$ & C & 2 \\
\hline 5'-GCACTTACCTGTGACTCCAT-3' & Verified & G & $A$ & 3 \\
\hline 5'-GGTCGTAACTGGCTCCATAG-3' & Verified & A & AT & 1 \\
\hline $5^{\prime}$-AAGCAGACAAGTCTAGCTGC- $3^{\prime}$ & Verified & G & $A$ & 1 \\
\hline 5'-GGAGAAAGTTGAGTGGCTTC-3' & Verified & G & $A$ & 1 \\
\hline
\end{tabular}

the whole exome samples. Frequent amplifications on chr8q, including $M Y C$; on chr20q, including ZNF217; and on chr17q, including ERBB2, HNF1beta, and STAT3, were observed in $64 \%, 54 \%$, and $46 \%$ of 39 samples, respectively. Other loci, including PIK3CA, KRAS, CCNE1, IL6, and IL6R were also amplified $(26 \%, 18 \%, 26 \%, 21 \%$, and $21 \%$ of samples, respectively). On the other hand, frequent deletions were observed on chr19p, including SMARCA4 (41\%); on chr13q, including $R B 1$ (28\%); on chr18q, including SMAD4 (21\%); and on chr9q, including NOTCH1 (21\%); the four loci that contained ARIDIA, ARIDIB, $S M A R C A 2$, and SMARCA4, which are components of the switch/sucrose nonfermentable (SWI/SNF) complex, were jointly deleted. ${ }^{7}$ Deletions were also found on chr17p where TP53 is located (Figure 3).
At the chromosome-arm level of resolution, amplified loci were found in both OCCC samples and GSE58342 at 1q, 3q, $7 \mathrm{p}, 8 \mathrm{p} / \mathrm{q}, 12 \mathrm{p}, 17 \mathrm{q}, 19 \mathrm{q}$, and 20p/q, and deletions were common to the two data sets at 4q, 9q, 11q, 13q, 18q, and 19p (GISTIC cutoff q-value <0.25) (Supplemental Table S2). Data set-specific amplifications and deletions were also detected (Supplemental Table S2). Genome-wide, the arm-level copy number alteration frequency correlations were highly significant between the two data sets at both amplified loci (Pearson $r=0.77, P<0.0001)$ and at deleted loci $(r=0.83$, $P<0.0001$ ) (Supplemental Table S2). At loci where intermittent copy number peak deviations were detected in the two data sets, deviation loci were discordant (GISTIC cutoff q-value <0.25) (Supplemental Figures S1 and S2). Although peak regions observed in both data sets do not precisely 


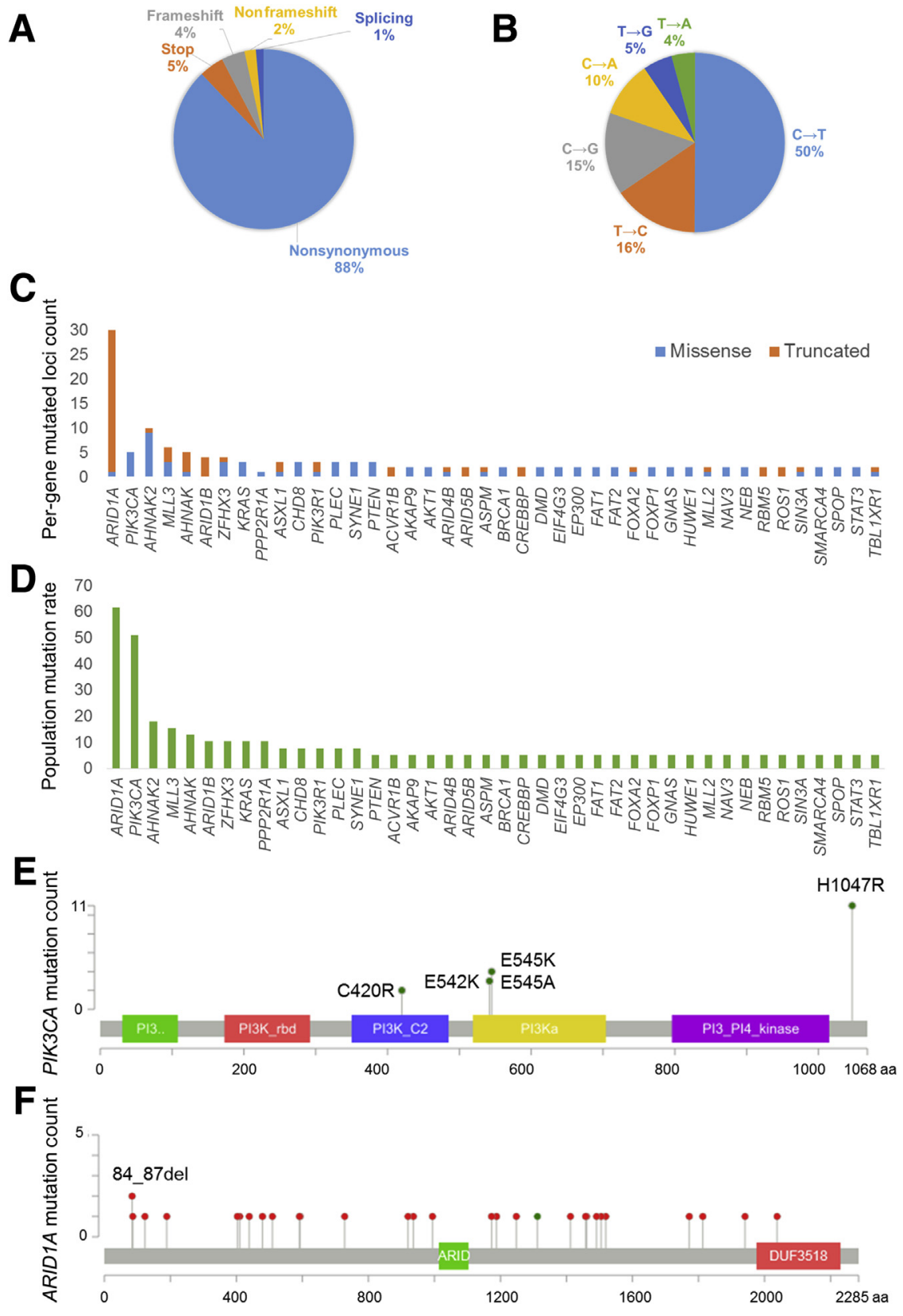

Figure 2 Spectra and distribution of ovarian clear cell carcinoma (OCCC)-associated somatic mutations. The frequency of nonsynonymous, truncation, and splicing variants in 3228 OCCCassociated somatic mutations $(\mathbf{A})$ and the nucleotide-level mutation spectrum of 3007 single-nucleotide mutations (B) are shown. The number of mutated loci for each gene (C) and the frequency of mutated genes (percentage of patients with at least one mutation present) in the 39 OCCC samples (D) are presented. Genes displayed are those from a prior pan-cancer study. ${ }^{23-25}$ For loci analysis, missense and truncating mutations were plotted in blue and orange bars, respectively. Mutated positions of the most frequently truncated gene ARID1A (E) and activated gene PIK3CA by nonsynonymous substitution $(\mathbf{F})$ are drawn with their functional domain structure. The number of missense and truncating mutations in each position are indicated with green and red circles, respectively.

correlate to one another, a variety of genes that are known to act as cancer drivers can be jointly observed to have mutation (GISTIC FDR q < 0.25) (Supplemental Figures S1 and S2).

We additionally examined the intra-data set copy number correlation between pairs of chromosome arms that contained genes that are hypothesized to exhibit a coordinated response. Despite correlations that are yielded via calculations, the correlations are spurious and did not yield actionable hypotheses (Supplemental Figures S3 and S4).

\section{Mutation Pathway and SNV-CNV Integrated Analysis}

The STRING database ${ }^{34}$ was used to map the 426 OCCCassociated genes containing multiple functional variants onto PPI data. Sixty-one genes were found to be involved a stringently defined PPI network (confidence score $>0.95$ ) (Figure 4 and Supplemental Table S3) and screened for significant enrichment in pathway-functional groups $(P<0.05)$ (Supplemental Table S4). As a result, two sufficiently sized networks with a total of 46 genes were annotated into four categorical groups that represented cancer biology, namely, cell proliferation, DNA repair and cell cycle checkpointing, chromatin remodeling (Figure 4A), and cytoskeletal organization (Figure 4B).

The network obtained recapitulates key known oncogenes, including, for example, PIK3CA, KRAS, and $P P P 2 R 1 A$ involved in cell proliferation. Members of the $\mathrm{SWI} / \mathrm{SNF}$ complex, including the critical gene ARIDIA, are 


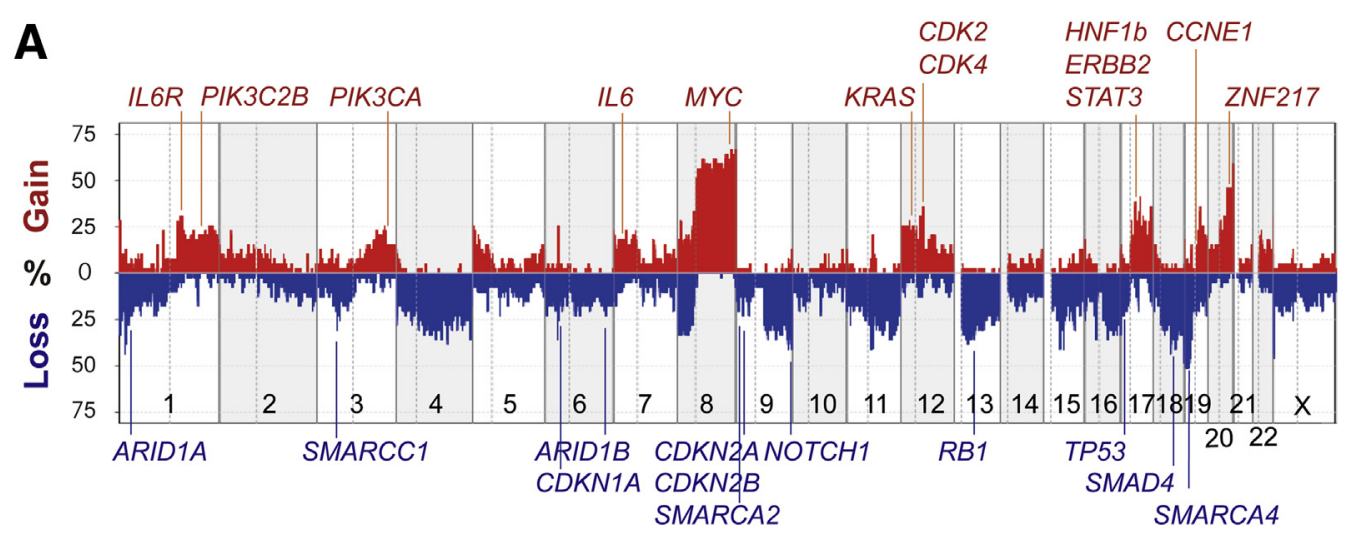

B

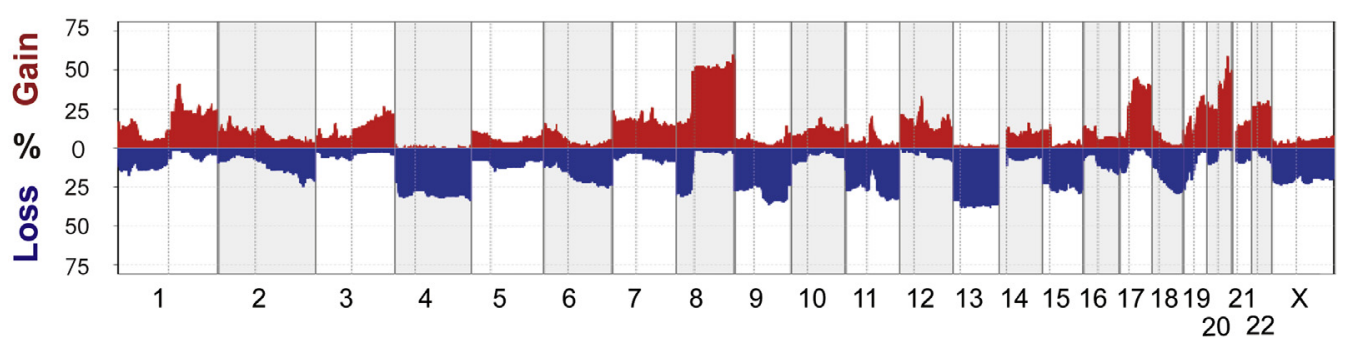

Figure 3 Copy number analysis of ovarian clear cell carcinomas (OCCC). Copy number alterations calculated from 39 exomes (A) and 112 array comparative genomic hybridization samples (https://www.ncbi.nlm.nih.gov/geo; accession number GSE58342) (B) of patients with 0CCC. The $x$ axis is the chromosomal position, and the $y$ axis is the mean copy number gain (red) or loss (blue) across each data set. Notable cancer-related genes located in the amplified or deleted loci are indicated by gene symbols with red and blue letters, respectively.

also in the network. Figure 4 gives a comprehensive view of how the pathways that contain highly mutated PIK3CA and ARIDIA are connected by multiple routes and which genes are the intermediates in those routes.

Key driver genes ARIDIA, PIK3CA, PIK3RI, and KRAS, among 19 total genes $(41 \%)$, were present in both the IntOGen and pan-cancer study gene list and our derived interaction network (Supplemental Table S5 and Supplemental Figure S5).

Integration of SNV and CNV analyses revealed that identified somatic mutations and CNVs were substantially enriched on the KRAS-PIK3CA-AKT1-PTEN pathway, the MYC-CDK2/ 4-RB1 pathway, and the aforementioned components of the SWI/SNF complex. These genetic alterations on the two pathways and remodeling complex were observed in $82 \%$, $79 \%$, and $85 \%$ of samples, respectively (Figure 5 ).

\section{Quality Assessment for Somatic Mutation Genes}

We checked whether matched tumor-normal pairs cases contained significantly different mutational landscape behavior then tumor-only samples (Supplemental Table S6). The result was negative (Fisher exact test). This finding reiterates our qualification of considering detected mutations to be somatic in nature.

The somatic mutations both in 16 paired cases and 23 unpaired cases contain ARIDIA, PIK3CA, ARIDIB, $A R I D 4 B, K R A S, P T E N, C R E B B P$, and PIK3Rl, which were also listed as part of the 19 common genes of our pathway analyses. Although PPP2R1A was found only in unpaired cases, it is reported to be a key somatic mutation gene in OCCC $^{10}$ (Supplemental Table S7).

Moreover, VAF in truncating and missense mutations was examined for the 426 candidate somatic mutation genes, visualized in Supplemental Figure S6. The VAFs are cross-checked against sequence read counts to warn against bias during handling. Neither inverse nor strong correlation was found in the measurements. The distribution of VAFs appeared to be roughly uniform. One difference that was detectable was the coverage (read counts) in genes with truncation mutations versus those with missense mutations (mean, 81.9 versus $95.0, P=0.002$ by $t$-test).

\section{Correlation between ARID1A Mutation and Its Protein Product BAF250a Expression}

The correlation between ARIDIA mutations with or without deletion and the expression of BAF250a, ARID1A's protein product, was confirmed by using immunoreactivity (Supplemental Figure S7) with per-patient analyses shown in Supplemental Table S8. For ARIDIA mutations with no deletions, a tendency for correlation with loss of expression in BAF250a was observed (Supplemental Figure S7A). After the inclusion of deletions, the association became statistically significant (Supplemental Figure S7B). Interestingly, of our 


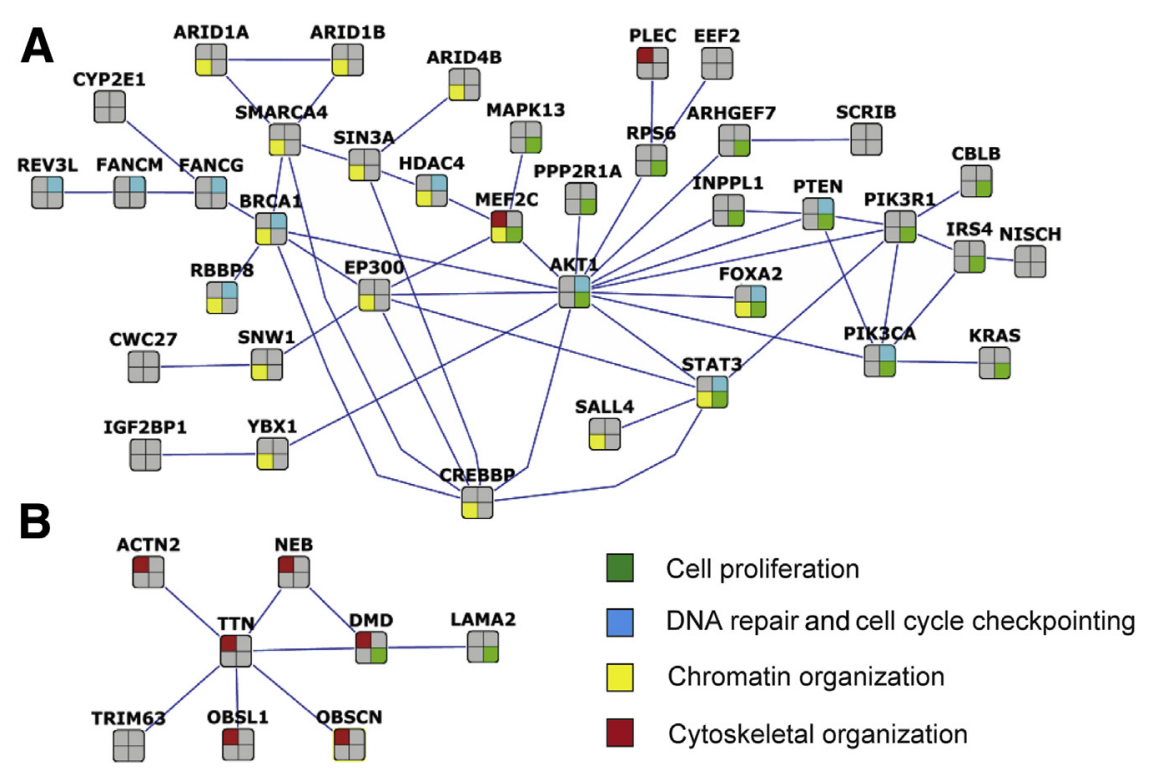

Figure 4 The connected landscape of somatic mutation in ovarian clear cell carcinoma (OCCC). Genes enriched in somatic mutations were crossreferenced against the STRING protein interaction database, and networks that included more than five genetic members were retained. Nodes are colored by significant functional pathways resulting from Database for Annotation, Visualization and Integrated Discovery analyses. Mutational events occurred at cancer-associated functional pathways. A: The network uncovers links between the proliferation and chromatin organization pathways. B: A separate, highly connected network of mutated cytoskeletal organization genes is also revealed.

39 cases, only two cases had a biallelic ARIDIA mutation. They demonstrated complete loss of BAF250a immunoreactivity. Obviously, a much larger patient population with biallelic mutation is required to substantiate this finding.

\section{PTEN Analysis}

Aside from mutation, PTEN expression loss is known to occur because of epigenetic silencing. ${ }^{40}$ Therefore, the possible routes of loss of expression are SNV, CNV, and epigenetic silencing, and because large numbers of SNVs and CNVs were not detected in our samples, results suggested epigenetic silencing was a factor. Experiments using immunohistochemistry revealed an $18 \%$ loss of expression of PTEN.

In our study, two cases with PTEN missense mutation had positive expression of PTEN IRS. One case with PTEN deletion had loss of expression of PTEN. Previous reports of PTEN loss of expression varied from $20 \%$ to $51 \%$, depending on the cutoff level used to evaluate the IRS. ${ }^{41,42}$

\section{Comparison of Copy Number by Exome Sequencing versus SNP Array}

For the subset of 12 OCCC samples with matching SNP arrays downloaded from Gene Expression Omnibus

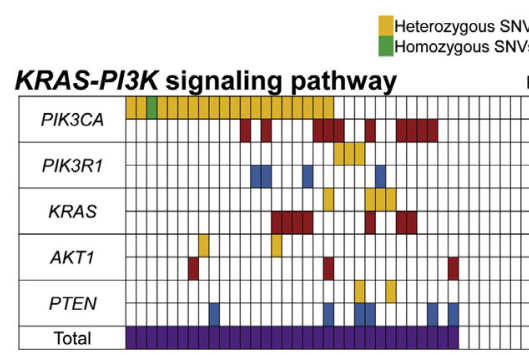

\section{MYC-RB signaling pathway}

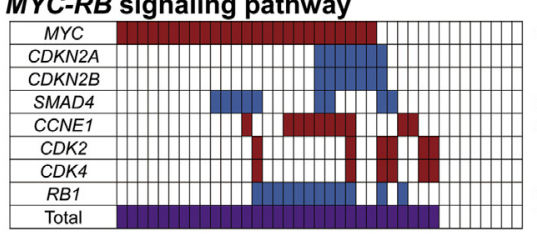

SWI/SNF complex

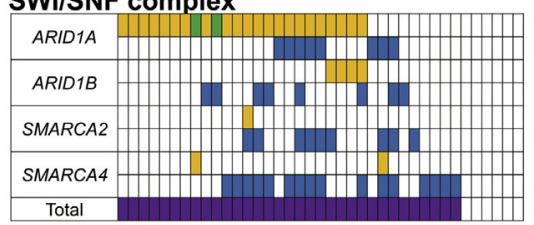

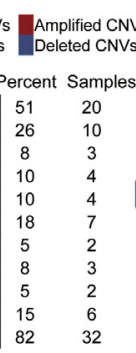

(n)

Cell proliferation, anti apoptosis

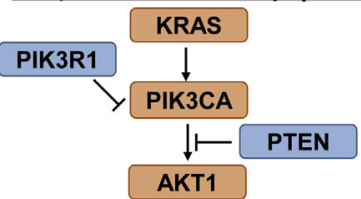

Cell cycle, $\mathbf{G 1} \rightarrow \mathbf{S}$

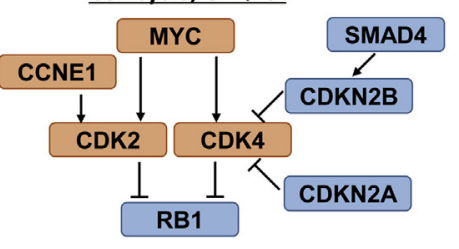

SWI/SNF complex
Figure 5 The integrated analysis of somatic mutations and CNVs. Heterozygous and homozygous somatic mutations and copy number amplification and deletion are represented with yellow, green, red, and blue, respectively. These genomic alterations are enriched in KRAS-PIK3CA-AKT1PTEN and MYC-CDK2/4-RB1 signaling pathways, as well as the ARID1A-ARID1B-SMARCA2-SMARCA4 components of the switch/sucrose nonfermentable (SWI/SNF) complex. 
(https://www.ncbi.nlm.nih.gov/geo; accession number GSE30311), matched array-exome samples were compared side by side for global similarity. Despite different underlying technologies and resolutions, the measurements are strongly correlated (data not shown).

\section{Discussion}

Full exome analysis of OCCC has been almost nonexistent thus far, and as a result of SNV, CNV, and combined analyses on a considerably sized sample, we have charted a first draft of the genome-wide events occurring in this ovarian cancer subtype. In the mutational spectrum of 3007 total base substitution mutations among 39 OCCC tumors, $\mathrm{C}$ to $\mathrm{T}$ and $\mathrm{T}$ to $\mathrm{C}$ substitutions were dominant (Figure $2 \mathrm{~B}$ ). It has been reported that such substitutions exhibit strong positive correlations with age in most cancer types. ${ }^{43}$

Four hundred twenty-six genes were identified that were repeatedly mutated in the OCCC samples. Carcinogenesis drivers ARIDIA (tumor suppression) and PIK3CA (oncogene) somatic mutations were observed in $62 \%$ and $54 \%$ of the OCCC samples, which is consistent with previous reports in human ${ }^{5,7,8}$ and mice. ${ }^{44,45}$ ARIDIA, belonging to a family of ATP-dependent chromatin remodelers known as the SWI/SNF complex, has been established as a tumor suppressor gene through the discovery of recurrent inactivating ARIDIA mutations in a broad spectrum of cancers, including endometriosis without cancer. ${ }^{46,47}$ Inactivating ARIDIA through somatic mutations and/or other epigenetic mechanisms results in loss of gatekeeper and caretaker functions in cells, thus promoting tumor initiation. ${ }^{48}$

The frequency of PPP2RIA somatic mutations (10\%) was also concordant with a separate targeted analysis in human endometrial and ovarian carcinomas. ${ }^{10}$ Interestingly, somatic mutations of PIK3CA and PIK3RI genes were mutually exclusive in our data set (Figure 5). To date, such an observation was reported for endometrial cancer $^{49}$ but not for OCCC.

$K M T 2 C / M L L 3$ and $A R I D 1 B$, related to chromatin modification and chromatin remodeling, respectively, were genes for which we identified somatic mutations in $15 \%$ and $10 \%$ of OCCC samples, respectively. These associations have not been previously reported. ${ }^{50}$ The mutations were truncations. Evidence of frequent mutations involved in chromatin remodeling and chromatin modification has accumulated in various types of cancer, and chromatin-associated mutations are thought to contribute to tumorigenesis via their effect on gene expression. ${ }^{50}$

The ARIDIA and ARIDIB genes have complementary roles to each other and have been reported to be comutated. $^{51,52}$ Mutation profiles obtained in this study contained co-mutation. This is in contrast to exclusive mutations detected for PIK3CA and PIK3RI (Figure 5).

We also detected somatic mutations in TTN, AHNAK2, and $A H N A K$ genes in 23\%, 18\%, and $10 \%$ of samples, respectively (Supplemental Table S1). These genes have been reported as frequently mutated and possibly driver genes in many cancers, ${ }^{25,50}$ but their functions are unclear. Because the TTN gene contains many repetitive sequences in its coding regions, the high frequency of somatic mutations of this gene is difficult to interpret. ${ }^{6}$ From the PPI network analysis, TTN has high-confidence interactions with six genes, containing characteristics of a hub gene (Figure 4). DAVID analysis revealed that TTN and four of its interaction partners are included in a 6-member actinbinding group (Supplemental Table S5); we consider TTN might play a role in tumor development through cell motility.

KRAS, PTEN, AKT1, FOXA2, STAT3, and EP300 were mutated in $5 \%$ to $7 \%$ of samples. These genes were located together on a $P I 3 \mathrm{~K}$ mitogenic signaling pathway in the protein-protein network map (Figure 4). Considering that infrequent mutations of multiple genes on the same pathway have been reported to lead to cancer proliferation, ${ }^{39,51-53}$ parallel mutations of these genes could also be important factors to develop OCCC. The network obtained has helped to connect the mitogenic cell proliferation pathway driven by $P I K 3 C A$ with the chromatin remodeling pathway driven by ARIDIA, expanding the number of possibilities in which infrequent mutations can combine to lead to the phenotype. The resulting network reconfirms our belief that PIK3CA is the accelerator for driving cell growth, with the braking mechanism provided by ARIDIA broken via mutation, combining to be the ideal pathway recipe for OCCC cancerization.

CNV analyses yielded a complementary dimension to the somatic mutation results. The CNV statuses obtained from the exome sequencing data were concordant with the signals from an external validation set of array CGH data (Figure 3). Previously, we reported that two CNVs occurred frequently in OCCC for array data: chr8q amplification and chr13q deletion, where oncogene $M Y C$ and tumor suppressor gene $R B 1$ are located, respectively. ${ }^{30}$ The results herein largely reproduce the previous report, ${ }^{30}$ indicating that exome sequencing has been substantially effective to detect not only somatic mutations but also CNVs from a single experimental data source. The high reproducibility of copy number aberration found in OCCC samples suggests that $\mathrm{CNVs}$ and somatic mutations are critical factors for carcinogenesis and progression of OCCC and may be useful in a joint, complementary fashion to characterize the behavior of an OCCC sample. Compared with a larger array CGH data set, the reproducibility and correlation of arm-level copy number alteration in OCCC exomes were observed (Supplemental Table S3). Although the reproducibility of CNV patterns was observed at arm levels and not at peak loci in the two independent data sets, peak loci nonetheless contained several cancer driver genes (ie, amplified loci contain oncogenes and deleted loci contain tumor suppressor genes). Data match previous reports that accumulation of alteration 
in cancer driver genes was observed sporadically (Supplemental Figures S1, S2, S3, and S4). ${ }^{13,54}$

Our integrated SNV/CNV analysis revealed that almost $80 \%$ of alterations occurred in the SWI/SNF complex, KRAS/phosphatidylinositol 3-kinase (PI3K) signaling, and MYC/retinoblastoma (RB) signaling pathways (Figure 5). In recent research involving genes included in these groups, an $\mathrm{EZH} 2$ inhibitor has been reported to be effective to treat cancer cells with ARIDIA (SWI/SNF) mutations. ${ }^{55}$ Similarly, PI3K and mechanistic target of rapamycin (mTOR) inhibitors (KRAS-PI3K) were beneficial for patients with cancer harboring PIK3CA alterations. ${ }^{56}$ The CDK2 inhibitor dinaciclib (MYC/RB) has recently begun evaluation for its effectiveness in treating ovarian tumors. ${ }^{57}$ Our results provide us with a possibility to target these pathways as an OCCC therapy, using methods such as chemogenomics $^{58-60}$ to leverage pathway and family similarity to rapidly identify inhibitors in recent or unpursued targets, such as $A R I D I B$ or $A H N A K$.

The equivalence of paired and unpaired cases is confirmed when considering mutation profiles in genes with low frequencies of mutation. However, when considering genes with high mutation frequencies (ARIDIA, PIK3CA, $A R I D I B, K R A S, P T E N$, and PIK3Rl), results were similar. Therefore, we thought our analyses with or without normal counterparts to be an overall reliable projection of the mutation landscape for OCCC.

Strong correlation between the loss of BAF250a protein expression and the presence of ARIDIA mutations has been reported in OCCC. ${ }^{7}$ In this study, we observed loss of BAF250a immunoreactivity in cases with ARIDIA mutations and deletions, although the sample size was limited (Supplemental Figure S7). Therefore, we can hypothesize that ARIDIA deletion might induce a loss of immunoreactivity in BAF250a. On the other hand, immunoreactive cases contained ARIDIA mutations, so some types of ARIDIA mutations do not seem to affect protein expression. An increased sample size might lead to clarification of association between ARIDIA mutation and BAF250a expression.

The current study has some limitation because of the unavailability of normal counterparts in some samples, which raises a possibility of inclusion of false germline mutations through the analysis. Nevertheless, we could successfully reproduce the identification of previously known genomic alterations. ${ }^{6}$ Furthermore, we also revealed novel associations of genomic alterations that occur frequently in the OCCC samples. The protein-product interaction network has generated a new perspective about how some functional groups with mutations are linked to each other by intermediaries (Figure 4). Taken together, this report yields additional evidence of the major factors in OCCC and targets for anticancer drug development.

OCCC is much more frequent in Japan compared with other populations and therefore deserves elevated research efforts. Planning is under way to increase the number of samples by collaborating with multiple centers of medical research throughout Japan. The effort is essential to deepen and expand knowledge about the subtype and to develop a tailored therapy driven by genetic diagnosis.

In conclusion, tumor-blood whole exome analyses reproduced mutations in known cancer genes and produced novel frequently mutated genes for further investigation. Independent SNV and CNV analyses, as well as their combination, provided insightful complementary views of the genome-wide landscape of OCCC. Elements of the KRAS/PI3K signaling pathway, the MYC/RB signaling pathway, and the SWI/SNF complex were enriched, reconfirming their status as potential therapeutic targets for patients with OCCC.

\section{Supplemental Data}

Supplemental material for this article can be found at http://dx.doi.org/10.1016/j.ajpath.2017.06.012.

\section{References}

1. Kurman RJ, Shih Ie M: The dualistic model of ovarian carcinogenesis: revisited, revised, and expanded. Am J Pathol 2016, 186 : $733-747$

2. Mandai M, Yamaguchi K, Matsumura N, Baba T, Konishi I: Ovarian cancer in endometriosis: molecular biology, pathology, and clinical management. Int J Clin Oncol 2009, 14:383-391

3. Sugiyama T, Kamura T, Kigawa J, Terakawa N, Kikuchi Y, Kita T, Suzuki M, Sato I, Taguchi K: Clinical characteristics of clear cell carcinoma of the ovary: a distinct histologic type with poor prognosis and resistance to platinum-based chemotherapy. Cancer 2000, 88: $2584-2589$

4. Itamochi H, Kigawa J, Terakawa N: Mechanisms of chemoresistance and poor prognosis in ovarian clear cell carcinoma. Cancer Sci 2008 , 99:653-658

5. Jones S, Wang TL, Shih Ie M, Mao TL, Nakayama K, Roden R, Glas R, Slamon D, Diaz LA Jr, Vogelstein B, Kinzler KW, Velculescu VE, Papadopoulos N: Frequent mutations of chromatin remodeling gene ARID1A in ovarian clear cell carcinoma. Science 2010, 330:228-231

6. Cancer Genome Atlas Research Network: Integrated genomic analyses of ovarian carcinoma. Nature 2011, 474:609-615

7. Wiegand KC, Shah SP, Al-Agha OM, Zhao Y, Tse K, Zeng T, et al: ARID1A mutations in endometriosis-associated ovarian carcinomas. N Engl J Med 2010, 363:1532-1543

8. Kuo KT, Mao TL, Jones S, Veras E, Ayhan A, Wang TL, Glas R, Slamon D, Velculescu VE, Kuman RJ, Shih Ie M: Frequent activating mutations of PIK3CA in ovarian clear cell carcinoma. Am J Pathol 2009, 174:1597-1601

9. Campbell IG, Russell SE, Phillips WA: PIK3CA mutations in ovarian cancer. Clin Cancer Res 2005, 11:7042; author reply-3

10. McConechy MK, Anglesio MS, Kalloger SE, Yang W, Senz J, Chow C, Heravi-Moussavi A, Morin GB, Mes-Masson AM, Carey MS, McAlpine JN, Kwon JS, Prentice LM, Boyd N, Shah SP, Gilks CB, Huntsman DG: Subtype-specific mutation of PPP2R1A in endometrial and ovarian carcinomas. J Pathol 2011, 223:567-573

11. Kuo KT, Mao TL, Chen X, Feng Y, Nakayama K, Wang Y, Glas R, Ma MJ, Kurman RJ, Shih Ie M, Wang TL: DNA copy numbers profiles in affinity-purified ovarian clear cell carcinoma. Clin Cancer Res 2010, 16:1997-2008 
12. Rahman MT, Nakayama K, Rahman M, Nakayama N, Ishikawa M, Katagiri A, Iida K, Nakayama S, Otsuki Y, Shih Ie M, Miyazaki K: Prognostic and therapeutic impact of the chromosome 20q13.2 ZNF217 locus amplification in ovarian clear cell carcinoma. Cancer 2012, 118:2846-2857

13. Kanchi KL, Johnson KJ, Lu C, McLellan MD, Leiserson MD, Wendl MC, Zhang Q, Koboldt DC, Xie M, Kandoth C, McMichael JF, Wyczalkowski MA, Larson DE, Schmidt HK, Miller CA, Fulton RS, Spellman PT, Mardis ER, Druley TE, Graubert TA, Goodfellow PJ, Raphael BJ, Wilson RK, Ding L: Integrated analysis of germline and somatic variants in ovarian cancer. Nat Commun 2014, 5:3156

14. Ciriello G, Miller ML, Aksoy BA, Senbabaoglu Y, Schultz N, Sander C: Emerging landscape of oncogenic signatures across human cancers. Nat Genet 2013, 45:1127-1133

15. Mabuchi S, Sugiyama T, Kimura T: Clear cell carcinoma of the ovary: molecular insights and future therapeutic perspectives. J Gynecol Oncol 2016, 27:e31

16. Yamaguchi K, Mandai M, Oura T, Matsumura N, Hamanishi J, Baba T, Matsui S, Murphy SK, Konishi I: Identification of an ovarian clear cell carcinoma gene signature that reflects inherent disease biology and the carcinogenic processes. Oncogene 2010, 29: $1741-1752$

17. Goodwin S, McPherson JD, McCombie WR: Coming of age: ten years of next-generation sequencing technologies. Nat Rev Genet 2016, 17:333-351

18. Higasa K, Miyake N, Yoshimura J, Okamura K, Niihori T, Saitsu H, Doi K, Shimizu M, Nakabayashi K, Aoki Y, Tsurusaki Y, Morishita S, Kawaguchi T, Migita O, Nakayama K, Nakashima M, Mitsui J, Narahara M, Hayashi K, Funayama R, Yamaguchi D, Ishiura H, Ko WY, Hata K, Nagashima T, Yamada R, Matsubara Y, Umezawa A, Tsuji S, Matsumoto N, Matsuda F: Human genetic variation database, a reference database of genetic variations in the Japanese population. J Hum Genet 2016, 61:547-553

19. Li H, Durbin R: Fast and accurate short read alignment with BurrowsWheeler transform. Bioinformatics 2009, 25:1754-1760

20. Li H, Handsaker B, Wysoker A, Fennell T, Ruan J, Homer N, Marth G, Abecasis G, Durbin R: The Sequence Alignment/Map format and SAMtools. Bioinformatics 2009, 25:2078-2079

21. McKenna A, Hanna M, Banks E, Sivachenko A, Cibulskis K, Kernytsky A, Garimella K, Altshuler D, Gabriel S, Daly M, DePristo MA: The Genome Analysis Toolkit: a MapReduce framework for analyzing next-generation DNA sequencing data. Genome Res 2010, 20:1297-1303

22. Wang K, Li M, Hakonarson H: ANNOVAR: functional annotation of genetic variants from high-throughput sequencing data. Nucleic Acids Res 2010, 38:e164

23. Gonzalez-Perez A, Perez-Llamas C, Deu-Pons J, Tamborero D, Schroeder MP, Jene-Sanz A, Santos A, Lopez-Bigas N: IntOGenmutations identifies cancer drivers across tumor types. Nat Methods 2013, 10:1081-1082

24. Gundem G, Perez-Llamas C, Jene-Sanz A, Kedzierska A, Islam A, Deu-Pons J, Furney SJ, Lopez-Bigas N: IntOGen: integration and data mining of multidimensional oncogenomic data. Nat Methods 2010, 7:92-93

25. Tamborero D, Gonzalez-Perez A, Perez-Llamas C, Deu-Pons J, Kandoth C, Reimand J, Lawrence MS, Getz G, Bader GD, Ding L, Lopez-Bigas N: Comprehensive identification of mutational cancer driver genes across 12 tumor types. Sci Rep 2013, 3:2650

26. Lawrence MS, Stojanov P, Polak P, Kryukov GV, Cibulskis K, Sivachenko A, et al: Mutational heterogeneity in cancer and the search for new cancer-associated genes. Nature 2013, 499:214-218

27. Cerami E, Gao J, Dogrusoz U, Gross BE, Sumer SO, Aksoy BA, Jacobsen A, Byrne CJ, Heuer ML, Larsson E, Antipin Y, Reva B, Goldberg AP, Sander C, Schultz N: The cBio cancer genomics portal: an open platform for exploring multidimensional cancer genomics data. Cancer Discov 2012, 2:401-404
28. Okamoto A, Sehouli J, Yanaihara N, Hirata Y, Braicu I, Kim BG, Takakura S, Saito M, Yanagida S, Takenaka M, Yamaguchi N, Morikawa A, Tanabe H, Yamada K, Yoshihara K, Enomoto T, Itamochi H, Kigawa J, Matsumura N, Konishi I, Aida S, Aoki Y, Ishii N, Ochiai K, Akiyama T, Urashima M: Somatic copy number alterations associated with Japanese or endometriosis in ovarian clear cell adenocarcinoma. PLoS One 2015, 10:e116977

29. Nilsen G, Liestol K, Van Loo P, Moen Vollan HK, Eide MB, Rueda OM, Chin SF, Russell R, Baumbusch LO, Caldas C, BorresenDale AL, Lingjaerde OC: Copynumber: efficient algorithms for single- and multi-track copy number segmentation. BMC Genomics 2012, 13:591

30. Huang RY, Chen GB, Matsumura N, Lai HC, Mori S, Li J, Wong MK, Konishi I, Thiery JP, Goh L: Histotype-specific copy-number alterations in ovarian cancer. BMC Med Genomics 2012, 5:47

31. Xi R, Hadjipanayis AG, Luquette LJ, Kim TM, Lee E, Zhang J, Johnson MD, Muzny DM, Wheeler DA, Gibbs RA, Kucherlapati R, Park PJ: Copy number variation detection in whole-genome sequencing data using the Bayesian information criterion. Proc Natl Acad Sci U S A 2011, 108:E1128-E1136

32. Lonigro RJ, Grasso CS, Robinson DR, Jing X, Wu YM, Cao X, Quist MJ, Tomlins SA, Pienta KJ, Chinnaiyan AM: Detection of somatic copy number alterations in cancer using targeted exome capture sequencing. Neoplasia 2011, 13:1019-1025

33. Mermel CH, Schumacher SE, Hill B, Meyerson ML, Beroukhim R, Getz G: GISTIC2.0 facilitates sensitive and confident localization of the targets of focal somatic copy-number alteration in human cancers. Genome Biol 2011, 12:R41

34. Szklarczyk D, Franceschini A, Wyder S, Forslund K, Heller D, Huerta-Cepas J, Simonovic M, Roth A, Santos A, Tsafou KP, Kuhn M, Bork P, Jensen LJ, von Mering C: STRING v10: proteinprotein interaction networks, integrated over the tree of life. Nucleic Acids Res 2015, 43:D447-D452

35. Smoot ME, Ono K, Ruscheinski J, Wang PL, Ideker T: Cytoscape 2.8: new features for data integration and network visualization. Bioinformatics 2011, 27:431-432

36. Warsow G, Greber B, Falk SS, Harder C, Siatkowski M, Schordan S, Som A, Endlich N, Scholer H, Repsilber D, Endlich K, Fuellen G: ExprEssence-revealing the essence of differential experimental data in the context of an interaction/regulation net-work. BMC Syst Biol 2010, 4:164

37. Abou-Taleb H, Yamaguchi K, Matsumura N, Murakami R, Nakai H, Higasa K, Amano Y, Abiko K, Yoshioka Y, Hamanishi J, Koshiyama M, Baba T, Yamada R, Matsuda F, Konishi I, Mandai M: Comprehensive assessment of the expression of the SWI/SNF complex defines two distinct prognostic subtypes of ovarian clear cell carcinoma. Oncotarget 2016, 7:54758-54770

38. Clevert DA, Mitterecker A, Mayr A, Klambauer G, Tuefferd M, De Bondt A, Talloen W, Gohlmann H, Hochreiter S: cn.FARMS: a latent variable model to detect copy number variations in microarray data with a low false discovery rate. Nucleic Acids Res 2011, 39:e79

39. Lawrence MS, Stojanov P, Mermel CH, Robinson JT, Garraway LA, Golub TR, Meyerson M, Gabriel SB, Lander ES, Getz G: Discovery and saturation analysis of cancer genes across 21 tumour types. Nature 2014, 505:495-501

40. Milella M, Falcone I, Conciatori F, Cesta Incani U, Del Curatolo A, Inzerilli N, Nuzzo CM, Vaccaro V, Vari S, Cognetti F, Ciuffreda L: PTEN: Multiple Functions in Human Malignant Tumors. Front Oncol 2015, 5:24

41. Wiegand KC, Hennessy BT, Leung S, Wang Y, Ju Z, McGahren M, Kalloger SE, Finlayson S, Stemke-Hale K, Lu Y, Zhang F, Anglesio MS, Gilks B, Mills GB, Huntsman DG, Carey MS: A functional proteogenomic analysis of endometrioid and clear cell carcinomas using reverse phase protein array and mutation analysis: protein expression is histotype-specific and loss of ARID1A/BAF250a is associated with AKT phosphorylation. BMC Cancer 2014, 14:120 
42. Friedlander ML, Russell K, Millis S, Gatalica Z, Bender R, Voss A: Molecular profiling of clear cell ovarian cancers: identifying potential treatment targets for clinical trials. Int J Gynecol Cancer 2016, 26 : $648-654$

43. Alexandrov LB, Nik-Zainal S, Wedge DC, Aparicio SA, Behjati S, Biankin AV, et al: Signatures of mutational processes in human cancer. Nature 2013, 500:415-421

44. Chandler RL, Damrauer JS, Raab JR, Schisler JC, Wilkerson MD, Didion JP, Starmer J, Serber D, Yee D, Xiong J, Darr DB, PardoManuel de Villena F, Kim WY, Magnuson T: Coexistent ARID1APIK3CA mutations promote ovarian clear-cell tumorigenesis through pro-tumorigenic inflammatory cytokine signalling. Nat Commun 2015, 6:6118

45. Guan B, Rahmanto YS, Wu RC, Wang Y, Wang Z, Wang TL, Shih Ie M: Roles of deletion of Arid1a, a tumor suppressor, in mouse ovarian tumorigenesis. J Natl Cancer Inst 2014, 106:dju146

46. Wu JN, Roberts CW: ARID1A mutations in cancer: another epigenetic tumor suppressor? Cancer Discov 2013, 3:35-43

47. Anglesio MS, Papadopoulos N, Ayhan A, Nazeran TM, Noe M, Horlings HM, et al: Cancer-associated mutations in endometriosis without cancer. N Engl J Med 2017, 376:1835-1848

48. Wu RC, Wang TL, Shih Ie M: The emerging roles of ARID1A in tumor suppression. Cancer Biol Ther 2014, 15:655-664

49. Kandoth C, Schultz N, Cherniack AD, Akbani R, Liu Y, Shen H, Robertson AG, Pashtan I, Shen R, Benz CC, Yau C, Laird PW, Ding L, Zhang W, Mills GB, Kucherlapati R, Mardis ER, Levine DA: Integrated genomic characterization of endometrial carcinoma. Nature 2013, 497:67-73

50. Gonzalez-Perez A, Jene-Sanz A, Lopez-Bigas N: The mutational landscape of chromatin regulatory factors across 4,623 tumor samples. Genome Biol 2013, 14:r106

51. Helming KC, Wang X, Roberts CW: Vulnerabilities of mutant SWI/SNF complexes in cancer. Cancer Cell 2014, 26:309-317
52. Watanabe R, Ui A, Kanno S, Ogiwara H, Nagase T, Kohno T, Yasui A: SWI/SNF factors required for cellular resistance to DNA damage include ARID1A and ARID1B and show interdependent protein stability. Cancer Res 2014, 74:2465-2475

53. Leiserson MD, Vandin F, Wu HT, Dobson JR, Eldridge JV, Thomas JL, Papoutsaki A, Kim Y, Niu B, McLellan M, Lawrence MS, Gonzalez-Perez A, Tamborero D, Cheng Y, Ryslik GA, Lopez-Bigas N, Getz G, Ding L, Raphael BJ: Pan-cancer network analysis identifies combinations of rare somatic mutations across pathways and protein complexes. Nat Genet 2015, 47: $106-114$

54. Hofree M, Shen JP, Carter H, Gross A, Ideker T: Network-based stratification of tumor mutations. Nat Methods 2013, 10:1108-1115

55. Bitler BG, Aird KM, Garipov A, Li H, Amatangelo M, Kossenkov AV, Schultz DC, Liu Q, Shih Ie M, Conejo-Garcia JR, Speicher DW, Zhang R: Synthetic lethality by targeting EZH2 methyltransferase activity in ARID1A-mutated cancers. Nat Med 2015, 21:231-238

56. Yap TA, Bjerke L, Clarke PA, Workman P: Drugging PI3K in cancer: refining targets and therapeutic strategies. Curr Opin Pharmacol 2015, 23:98-107

57. Chen XX, Xie FF, Zhu XJ, Lin F, Pan SS, Gong LH, Qiu JG, Zhang WJ, Jiang QW, Mei XL, Xue YQ, Qin WM, Shi Z, Yan XJ: Cyclin-dependent kinase inhibitor dinaciclib potently synergizes with cisplatin in preclinical models of ovarian cancer. Oncotarget 2015, 6: 14926-14939

58. Bredel M, Jacoby E: Chemogenomics: an emerging strategy for rapid target and drug discovery. Nat Rev Genet 2004, 5:262-275

59. Brown JB, Niijima S, Okuno Y: Compound protein interaction prediction within chemogenomics: theoretical concepts, practical usage, and future directions. Mol Inform 2013, 32:906-921

60. Reker D, Schneider P, Schneider G, Brown JB: Active learning for computational chemogenomics. Future Med Chem 2017, 9:381-402 